\title{
Propuesta de intervención educativa para la prevención de la conducta suicida en adolescentes en la ciudad de Manizales (Colombia)*
}

\author{
Educational intervention proposal for the prevention of \\ suicidal behaviour in adolescents in Manizales city (Colombia)
}

\author{
Sandra Constanza Cañón \\ ORCID 0000-0001-7228-2420 \\ José Jaime Castaño Castrillón ** \\ ORCID 0000-0002-2300-4990 \\ Andrea Marcela Mosquera Lozano \\ ORCID 0000-0003-1614-7587 \\ Luisa María Nieto Angarita \\ María Orozco Daza \\ William Felipe Giraldo Londoño \\ Universidad de Manizales, Colombia \\ Recibido: 20 de enero de 2017 \\ Revisado: 1 de abril de 2017 \\ Aceptado: 2 de junio de 2017
}

\section{Resumen}

El objetivo de este estudio fue realizar una intervención educativa para la prevención de la conducta suicida en adolescentes entre los grados $6^{\circ}$ a $8^{\circ}$ de un colegio de la ciudad de Manizales, en el departamento de Caldas, Colombia. Se realizó un estudio cuasi experimental, que contó con una población de 103 participantes. Se cuantificaron en la población variables demográficas. Adicionalmente se aplicaron la escala de Riesgo Suicida de Plutchik, la encuesta mito o realidad, escala de Asertividad de Rathus, y escala de autoestima de Rosenberg. Seguidamente se realizó una intervención educativa en 8 sesiones, con los estudiantes participantes divididos en 4 grupos. Un mes después se aplicaron nuevamente los cuestionarios mencionados. En la aplicación de los cuestionarios, antes de la intervención educativa, se encontró un $42,7 \%$ de riesgo suicida, en mayor proporción $56,9 \%$ de autoestima baja, en mayor proporción $68,9 \%$ de asertividad confrontativa, y 2,9 en

Artículo de investigación. http://dx.doi.org/10.15332/s1794-9998.2018.0001.02

Correspondencia: José Jaime Castaño Castrillón, Orcid 0000-0002-2300-4990, Profesor Titular, Grupo de Psicología Clínica y Procesos de Salud. Facultad de Ciencias de la Salud, Universidad de Manizales, Dirección postal: Carrera 9¹9-03, Manizales, Caldas, Colombia. Correo electrónico: jcast@umanizales.edu.co; sandraka@umanizales.edu.co 
el cuestionario sobre mitos alrededor del suicido. Después de esta intervención se encontró: factor de riesgo suicida de $6,8 \%$, en mayor proporción autoestima alta en $45,1 \%$, en mayor proporción $44,7 \%$ presentaron asertividad confrontativa, y 4,5 en el cuestionario sobre mitos. La prueba t para grupos apareados mostró en todos los casos un aprendizaje significativo $(p<0.001)$. Como conclusión, la investigación resaltó la importancia de que los jóvenes reconocieran y fortalecieran las habilidades sociales, tengan comunicación asertiva y destrezas intelectuales con el fin de reducir el riesgo suicida en la población estudiantil.

Palabras clave: autoestima, adolescente, prevención primaria, suicidio, educación.

\section{Abstract}

Objective: To assess the effect of an educational intervention to prevent suicidal behavior in teenagers of grades 6th to 8th at a school in Manizales, Colombia. Materials and methods: Quasiexperimental study $(n=103)$, that collected demo-graphic variables, the Plutchik Suicide Risk Scale, the Myth or Reality Survey, the Rathus Assertiveness Scale, and the Rosenberg Self-Esteem Scale. Participants were divided into five groups and an educational intervention lasting 8 sessions was given to them. A month later, the same questionnaires were administered. Results: Before the educational intervention, suicidal risk was identified in $42.7 \%$ of the sample, low self-esteem in $56.9 \%$, confrontational assertiveness in $68.9 \%$, and 2,9 on the myths around suicide questionnaire. After the interven-tion, the suicide risk factor fell to $6.8 \%$, high self-esteem was found in $45.1 \%$, a greater proportion of patients, $44.7 \%$ showed confrontational assertiveness, and the score on the myths questionnaire rose to 4.5. The t-test for paired groups showed significant learning in all cases $(p<0.001)$. Conclusions: The study highlighted the importance recognizing and strengthening social skills, having assertive communication and intellectual skills in order to reduce suicidal risk in young students.

Keywords: self-esteem, adolescent, primary prevention, suicide, education

\section{Introducción}

El suicidio es un fenómeno de causa multifactorial en el que intervienen factores biológicos, psicológicos, sociales, culturales y ambientales (Herrera $\&$ Betancour, 2012, Ruiz, 2009), este a su vez puede considerarse como la expresión de una dificultad en los mecanismos adaptativos del sujeto al medio ambiente provocado por una situación de conflicto que genera un estado de tensión emocional. Durkheim (1992) define el suicidio como "todo caso de muerte que resulta directa o indirectamente de un acto positivo o negativo realizado por la víctima misma, y que, según ella sabía, debía producir este resultado" (Durkheim, 1992, p. 16) El suicidio a su vez es un proceso que va desde una ideación suicida pasiva, seguida por fases como la visualización del acto y su presentación mediante amenazas verbales o gestos suicidas, planeación, preparación, ejecución del acto, y finalmente el suicido consumado.

Actualmente el suicidio es un fenómeno social con una alta tasa de mortalidad, según cifras de la OMS (OMS, 2012) más de 800.000 personas se suicidan cada año, y se considera como la segunda causa principal de defunción en el grupo etario de 15 a 29 años; se ha establecido además que aproximadamente el $75 \%$ de todos los suicidios se producen en países de ingresos bajos y medianos (OMS, 2012; Ruiz, 2009).

Cabe destacar que un intento de suicidio no consumado se convierte en el factor de riesgo más importante para suicidio. De acuerdo a cifras globales reveladas por la OMS, el suicidio aportó el 
$1,8 \%$ de morbilidad para la década del 90 (OMS, 2012), y se prevé que para el 2020 representará el 2,4\% de muertes en algunos países como Alemania, la Unión Soviética, China, Corea del Norte, Estados Unidos entre otros (OMS, 2014).

De otro lado en Colombia, el informe de Seguridad de la Veeduría Distrital de Bogotá registró que el mayor porcentaje de suicidio está representado en el género masculino, siendo un $89 \%$, de los cuales 22 casos fueron en adolescentes y jóvenes y el resto en la población adulto (Nope, 2015). Para el 2011 en Bogotá la cifra de suicidio consumado estableció que, por cada 5 hombres, 1 mujer se quita la vida para un total de 277 suicidios en este año (Macana, 2011).

También los diferentes grupos etarios son afectados por diversos factores de riesgo como: pensamientos suicidas, depresión, embarazos a temprana edad, violencia intrafamiliar, familias disfuncionales, pobreza extrema, pocas oportunidades, consumo de sustancias psicoactivas, entre otros, que conllevan a "suicidios consumados como resultado final” (Gutiérrez, 2010).

Dado que el suicidio es un problema importante en los adolescentes se hace necesaria su prevención, enfocada principalmente en la observación e identificación de las diferentes señales del comportamiento suicida entre los adolescentes (Vásquez, 2012). Con frecuencia, las conductas suicidas de los jóvenes pasan inadvertidas por los padres y los profesionales de la salud. Es preocupante que, aunque estos índices sean considerados a nivel mundial como un problema de salud pública (OMS, 2012; Instituto Nacional de estadísticas y geografía de México, 2015), el 60\% de las personas con pensamientos suicidas no recibe ningún tipo de ayuda profesional (Pitman, Osborn, King \& Erlangsen, 2014).

El aspecto más importante de la prevención del suicidio es el reconocimiento de los jóvenes en situaciones de aflicción y/o con un alto riesgo de suicidio (Ventura, et al., 2010); motivo por el cual las escuelas y los colegios se convierten en el escenario perfecto para prevenir estas conductas; por esto los programas educativos han adquirido un gran auge con el fin de aumentar la sensibilización y el conocimiento acerca del suicidio, conductas de riesgo, estrategias de prevención y signos de alarma. Uno de los principales objetivos de los profesionales en salud es disminuir esta tasa mediante la aplicación de estrategias de promoción y prevención en materia de salud mental (Vargas, Vásquez, Soto \& Ramírez, 2015).

La implementación de estrategias de promoción y prevención ha hecho evidente una notoria reducción en las cifras de dicha problemática: como por ejemplo el taller "Salvando Vidas" realizado en el 2008, aplicado en ocho preparatorias de la Universidad de Guanajuato, México (Chávez, Medina \& Macías, 2008), aportó conocimientos valiosos sobre los factores desencadenantes y conductas inapropiadas entre los jóvenes, planteando una estrategia de intervención psicoeducativa, enfocada en los factores de riesgo y los factores protectores, identificando en ambos los conocimientos que tenían previamente los jóvenes participantes por medio de un cuestionario. Este estudio arrojó como resultado la abolición de mitos en torno al suicidio. Además, se demostró que los participantes del estudio corrigieron y complementaron sus creencias acerca de esta condición. Otro ejemplo de este tipo de estrategias de promoción y prevención es el estudio realizado en el 2014 llamado taller "Surviving the teen", aplicado a estudiantes de educación en The Greater Cincinnati School, (King, Strunk, \& Sorter, 2011), allí se logró demostrar la efectividad de esta estrategia, puesto que los participantes estuvieron durante tres meses realizando talleres enfocados en pensamientos positivos; se logró analizar que durante este tiempo fueron menos propensos a comportamientos de riesgo, logrando una construcción positiva en la búsqueda de ayuda y mayor acercamiento a un adulto de confianza con el cual pudieran compartir sus situaciones y problemas.

La importancia de este tipo de programas de prevención sobre suicidio en jóvenes permite ayudarlos a adquirir competencias para reconocer los problemas, alcanzar habilidades para solucionarlos, identificar redes de apoyo, ampliando su bagaje de respuestas ante condiciones adversas; logrando que ellos utilicen los aprendizajes que se les imparten en estos programas. Buscan la 
consolidación de la autoeficacia, las herramientas sociales y las actitudes de cambio, provocando una mejora del carácter ante la confrontación de las situaciones de crisis.

También es relevante lograr que la población en formación omita todas aquellas creencias y mitos acerca del suicidio, recreando más espacios para la socialización y discusión con los estudiantes, suministrando la información precisa sobre las señales y síntomas del suicidio, reforzando los factores de protección para disminuir los factores de riesgo que incidan en el aumento de conductas suicidas, teniendo en cuenta de forma simultánea, factores psicológicos, el medio circundante, el ambiente económico y el ámbito sociocultural en el cual se encuentran las jóvenes, así, los estudiantes estarán más preparados para solicitar ayuda para sí mismos o para sus amigos.

Por todo lo anterior el propósito de esta investigación fue realizar una intervención psicoeducativa para prevenir la conducta suicida en los estudiantes de un colegio de secundaria en la ciudad de Manizales, Caldas, basado en la detección temprana de señales de advertencia en grupos vulnerables, así como la promoción de factores protectores en una población específica y la educación a jóvenes. Esta intervención les permitiría a los jóvenes adquirir competencias en autoestima, resolución y manejo tanto de conflictos socioculturales como de problemas interpersonales, y que, además, puedan descubrir la capacidad que posee cada joven de subsanar rápidamente sus problemas, cultivar amistades y relacionarse con su entorno, ofreciéndoles herramientas para la resolución de adversidades y fortalecimiento de las habilidades sociales. Todo esto con el apoyo de elementos audiovisuales, y actividades como la danza, la lectura, el deporte, el arte, conversatorios y todas aquellas actividades que contribuyan a la solución de problemas y convivencia sana en sociedad (Henao, López \& Mosquera, 2014).

\section{Métodos}

Investigación con estudio cuasi experimental. La población se conformó por 103 participantes entre los grados $6^{\circ}$ a $8^{\circ}$ de un colegio de secundaria de la ciudad de Manizales (Colombia), que diligenciaron el consentimiento informado con los padres de familia.

Las variables que se cuantificaron en la población de estudio fueron: la edad, género, estrato socioeconómico, tipo de familia, año escolar y práctica religiosa. Adicionalmente, se aplicaron la escala de Riesgo Suicida de R. Plutchik (Plutchik, 1989; Rubio, et al.,1998) para identificar factor de riesgo suicida (FR suicida), encuesta mito o realidad, escala de Asertividad de Rathus (León, 2009) para determinar la asertividad y Escala de autoestima de Rosenberg (Martínez, Trujio, Díaz \& Osma, 2011) para valorar la autoestima.

\section{Instrumentos}

\section{Escala de Riesgo suicida de Plutchik}

El riesgo suicida se evaluó mediante este cuestionario, donde los factores de estudio son la ideación e intento suicida, sentimientos de depresión y desesperanza e impulsividad, y se tiene en cuenta que a mayor puntaje mayor será el riesgo suicida, partiendo de 6 como punto de corte. La escala fue diseñada por Robert Plutchik con el objeto de discriminar a los pacientes suicidas de aquellos que no lo son. La versión española fue validada por Rubio, et al. (1998). Evalúa intentos suicidas previos, intensidad de la ideación suicida actual, sentimientos de depresión y desesperanza. La escala original muestra una consistencia de 0,84 (alfa de Cronbach) y en la validación española el alfa de Cronbach alcanzó valores de 0,90.

\section{Escala de autoestima de Rosenberg (1965)}

El nivel de autoestima se midió mediante esta escala, que consta de 10 afirmaciones de los sentimientos que tiene la persona sobre ella, 5 direccionadas positivamente (ítems $1,2,4,6$ y 7) y 5 negativamente (ítems 3, 5, 8, 9 y 10), de esta manera los valores teóricos fluctúan entre $10 \mathrm{y}$ 40; quien se encuentran cerca de 10 tendrá baja autoestima y a 40 alta autoestima. Esta escala se trabajó con la validación chilena realizada por Rojas, Zegers y Forster (2009), y la validación en 
español fue realizada por Atienza, Moreno y Balaguer (2000).

\section{Escala dimensional del sentido de la vida (Martínez, et al., 2011; Oiva, 2014)}

Esta escala tiene por objeto valorar la percepción afectivo-cognoscitiva de valores referenciales que mueven a una persona y le invitan a actuar de un modo particular ante situaciones específicas o la vida en general, confiriéndole de identidad personal y coherencia consigo misma. La escala dimensional del sentido de la vida se encuentra validada en Colombia por Martínez, et al. (2011).

\section{Cuestionario de asertividad de Rathus}

El cuestionario tiene como objetivo evaluar conductas relacionadas con la expresión de opiniones, peticiones o negación ante determinadas situaciones sociales. El instrumento está diseñado para ser auto administrado, de tal forma que los sujetos evalúan cada afirmación de acuerdo con una escala de seis anclajes, la cual va de -3 (muy poco característico de mí, extremadamente no descriptivo) a +3 (muy característico de mí, extremadamente descriptivo). La puntuación total oscila entre -90 y 90 , siendo mayor cuanto mayor es la asertividad. Este instrumento ha sido valido en Colombia por Londoño \& Valencia (2008).

\section{Procedimiento}

Se diseñó un proceso que se desarrolló en 8 sesiones que se describen a continuación:

\section{Sesión I: Consentimiento informado.}

Se realizó el consentimiento informado con los estudiantes que participaron en la investigación, para esto se les explicó la metodología de la investigación, se dejó claro todo tipo de implicación ética o de otra índole y se aclararon dudas con respecto a la investigación, posteriormente se les entregó un formato que diligenciaron con la aprobación de cada acudiente.

\section{Sesión II: Aplicación de escalas y cuestionarios}

Se comenzó con la aplicación de una serie de cuestionarios que cada alumno respondió de forma individual, estos correspondieron a la escala de riesgo suicida de Plutchik, escala de autoestima de Rosenberg, escala dimensional de sentido de la vida y cuestionario sobre comunicación asertiva; los cuales se evaluaron de acuerdo a la calificación de cada escala. La duración fue de unos 60-120 minutos.

\section{Sesión III: Cuestionario "mito o realidad"}

Los mitos son criterios culturalmente aceptados y entronizados en la población que no reflejan la veracidad científica, pues se trata de juicios de valor erróneos con respecto al suicidio, a los suicidas y a los que intentan el suicidio, que deben ser eliminados si se desea colaborar con este tipo de personas.

En este taller cada integrante recibió un cuestionario con 26 enunciados acerca de los mitos sobre suicidio, cada ítem lo respondieron con "Falso" o "Verdadero" según el conocimiento de cada integrante. Posteriormente se les realizó una socialización de cada enunciado y se les brindó un verdadero criterio científico acerca de cada mito, y se aclararon todas las dudas respecto al tema. La duración de esta sesión fue de unos 60-90 minutos.

\section{Sesión IV}

Se realizaron una serie de actividades entorno a la autoestima, desarrolladas en 3 módulos, cada uno con una duración aproximada de 60 minutos:

\section{Taller, ¿Quién soy y como me gustaría ser?}

En este módulo se trató el tema del conocimiento de sí mismo a través de la búsqueda de aspectos positivos y negativos referentes a la imagen física, a la forma de ser, de comportarse e identificaron aquello que los hace únicos y diferentes al resto. 
Ser consciente de lo que se hace, de lo que se está aprendiendo, de lo que queda por aprender, es decir, lo que es cada uno (yo real) y lo que les gustaría ser (yo ideal).

Actividades: Cada joven construyó en un papel su silueta, colocando dentro de ella cómo se define, "yo real", y fuera de la misma aquello que ha de cambiar o le gustaría tener, objetivos por los que se está esforzando, situando más próximo a su cuerpo lo más fácil de conseguir y a mayor distancia lo que le puede resultar más difícil o más lejano en el tiempo, lo que representaría el "yo ideal".

\section{Sesión $V$}

1. Taller Otros significativo (Salgado, 2011): Tuvo como objetivo identificar a las personas significativas para cada participante, tomando conciencia acerca de por qué y para quién es importante, qué valoran los demás de él (ella), qué valora él de los demás y qué le piden los demás para mejorar sus relaciones con ellos.

Actividades: cada joven construyó varios círculos alrededor del suyo, colocando en los círculos más próximos a las personas más importantes para él (ella) dentro del grupo. Al finalizar se les pidió que reflexionaran y dijeran la causa por la cual ellos consideran importantes a estas personas.

2. Taller ¿Qué hago yo para merecer esto? (González \& Gimeno, 2013): Este módulo hace referencia a las atribuciones causales de los éxitos, fracasos y de los sucesos positivos y negativos que ocurren en su vida. Que buscó identificar los logros y los aspectos que valoran ellos y los otros en positivo y en negativo -aspectos trabajados en los dos primeros módulos- se les pidió que identificar las razones y los responsables de los mismos, de sus conductas y de por qué consiguen o no un objetivo.

Actividades: al joven se le presenta una cartulina con dos columnas deno-minadas "Yo soy responsable de..." y "Son los otros responsables de...". En ellas colocaron las características físicas, psicológicas, conductuales y objetivos positivos y negativos que tienen. Luego reflexionaron en grupo acerca de las mismas, pretendiendo tomar conciencia que las cualidades positivas dependen de uno mismo.

\section{Sesión VI: Terapia a través del arte}

Se realizó otra actividad entorno a la comunicación asertiva, la cual tuvo una duración aproximada de 1 hora:

\section{Terapia a través del arte}

El arte refleja las tendencias internas de la sociedad y es el ámbito idóneo para el desarrollo de la expresión personal y de la comunicación, consigo mismo y con los demás. La terapia a través del arte es una forma de psicoterapia que trata los padecimientos emocionales ayudando al participante a comunicar sus pensamientos y sentimientos de manera gráfica, a través del dibujo, la pintura, el modelado u otras artes plásticas. Este taller se llevó acabo por medio del test del árbol (Rodríguez \& Castaño, 2002), es uno de los instrumentos proyectivos más simples de efectuar por parte de niños, jóvenes y también adultos.

Detrás de lo simple del árbol van apareciendo plasmados en el papel los diferentes elementos básicos que configuran la estructura del propio "yo". El árbol toma la personalidad del autor y deja contemplar la riqueza de su paisaje personal y sus matices. Es un lienzo único que refleja la verdadera esencia de quien lo ha dibujado.

\section{Sesión VII: Expresión corporal}

Se realizó una serie de actividades entorno a la comunicación asertiva, las cuales tuvieron una duración aproximada de 1 hora:

Biodanza (Castañeda, 2014): En este ítem se resalta aquello que se vive a través del baile, desarrollando habilidades corporales que ayudan al fortalecimiento la autoestima, proyección de sus pensamientos y liberación de sus emociones, desarrollo de potencialidades humanas mediante la vivencia integrada y la música. 
En la propuesta de biodanza se puso en contacto las emociones a través de la comunicación con el cuerpo, el movimiento y la música. Se realizó la sesión por grupos de 25 estudiantes guiada por una psicóloga con experiencia en artes escénicas, donde éstos estuvieron en contacto con las emociones a través de la comunicación con el cuerpo, el movimiento y la música.

Se observó desarrollo de la espontaneidad y desinhibición de los estudiantes. Cada estudiante recordó el momento más triste de su vida y la forma como lo resolvieron, mientras sus compañeros les planteaban diferentes formas positivas de cómo solucionar las dificultades.

\section{Sesión VIII: pos-test}

En esta sesión se realizó el postest, en el cual cada integrante desarrolló nuevamente las escalas y cuestionarios indicados en la investigación.

En el periodo comprendido en el mes de septiembre del año 2015 se realizó la primera y segunda sesión aplicada a un total de 103 participantes divididos en 4 grupos cada uno conformado por: 3 grupos de 26 estudiantes y 1 grupo de 25 estudiantes. En el mes de octubre se realizó la tercera, cuarta y quinta sesión; la sexta sesión se realizó en febrero del 2016; en el mes de marzo del mismo año se llevó a cabo la séptima sesión la cual se dividió en dos fechas tomando la mitad de los participantes que fueron 51 estudiantes y estos se subdividieron en dos grupos, uno de 26 y otro de 25; a la semana siguiente se realizó la misma sesión a la mitad restante subdividiéndolos en dos grupos de 26 estudiantes cada uno; la última sesión se realizó a principios del mes de abril de 2016. En cada sesión siempre participaron dos miembros del grupo de investigación.

Referente a los procedimientos estadísticos empleados, las variables medidas en escala nominal se describieron mediante tablas de frecuencia y límites de confianza al 95\%. Las variables medidas en escala numérica mediante promedios, desviaciones estándar, y límites de confianza al 95\%. Las diferencias entre el pre y pos-test se midieron mediante la prueba t pareada. Las relaciones entre variables medidas en escala nominal se probaron mediante la prueba de $\mathrm{x}^{2}$. Todos los análisis de inferencia estadística se hicieron con una $a=0,05$. La base de datos se elaboró con el programa Excel 2013 (Microsoft Corp), y se analizó mediante el programa estadístico IBM SPSS 22 (IBM Corp).

El proyecto fue enviado a la institución educativa para aprobación y comentarios. Los acudientes de los estudiantes firmaron un consentimiento informado, y en todo momento se respetó el anonimato de ellos.

\section{Resultados}

Intervinieron en el estudio 103 estudiantes. Las variables demográficas de esta población y las correspondientes al proceso se encuentran detalladas en la Tabla 1. Allí se observa que el 53,9\% de la población pertenece al género femenino. $\mathrm{El}$ $37,4 \%$ de la población pertenece a un estrato socioeconómico 2, con una edad promedio de 14,78 años (IC 95\%:14,5-15). El 86,3\% tienen práctica religiosa, de los cuales el 78,6\% practican la religión católica. El 46,1\% de los estudiantes tiene un tipo de familia nuclear (Ver Tabla 1).

Antes del proceso educativo los cuestionarios aplicados tuvieron los siguientes resultados: Factor de riesgo suicida positivo según cuestionario de Plutchick: 42,7\% (IC 95\%:34\%-50,5\%), según el cuestionario de autoestima de Rosenberg la mayor proporción 56,9\% (IC 95\%:48\%-65,7\%) tenían autoestima baja, según la escala dimensional del sentido de la vida de Rathaus el 68,9\% (IC 95\%: 61,2\%-77,3\%) presentaron asertividad confrontativa. Referente al cuestionarlo mito o realidad, que muestra ideas acertadas sobre el suicidio el promedio obtenido fue de 2,9 (IC 95\%: 2,69-3,03) donde 5 representa las ideas más acertadas. (Tabla 1).

Después del proceso de enseñanza, en la aplicación de cuestionarios realizada un mes después del proceso, los resultados obtenidos fueron: factor de riesgo suicida positivo de $6,8 \%$ (IC 95\%: 2,9\%-10,7\%), en mayor proporción autoestima alta en 45,1\% (IC 95\%: 37,2\%-52,5\%), en mayor proporción 44,7\% (IC 95\%: 35,9\%-53.4\%) presentaron 
asertividad confrontativa. La escala dimensional del sentido de la vida presentó un promedio de 2,85 (IC 95\%: 2,79-2,02). El cuestionario mito o realidad presentó un resultado de 4,5 (IC 95\%: 4,45-4,65). (Tabla 1).

El cuestionario de Plutchick presentó una a de Cronbach de ,826, el de Rosenberg de ,876, el de Rathaus de ,577, la escala dimensional del sentido de la vida ,418 y el cuestionario mito o realidad de ,899.

Tabla 1.

Variables demográficas, y resultado de cuestionarios de la población de estudiantes de secundaria que participó en el estudio.

\begin{tabular}{|c|c|c|c|}
\hline Variable & Niveles & $\mathrm{N}$ & $\%$ \\
\hline \multirow{3}{*}{ Género } & Femenino & 55 & 53,9 \\
\hline & Masculino & 47 & 46,1 \\
\hline & Faltantes & 1 & 1 \\
\hline \multirow{5}{*}{$\begin{array}{l}\text { Estrato } \\
\text { Socioeconómico }\end{array}$} & 2 & 37 & 37,4 \\
\hline & 3 & 35 & 35,4 \\
\hline & 1 & 25 & 25,3 \\
\hline & 4 & 2 & 2,0 \\
\hline & Faltantes & 4 & 3,9 \\
\hline \multirow{4}{*}{ Edad } & Promedio & 14,78 & \\
\hline & Des. Est. & 1,3 & \\
\hline & Ic $95 \% \mathrm{Li}$ & 14,5 & \\
\hline & Ic $95 \%$ Ls & 15 & \\
\hline \multirow{9}{*}{ Práctica Religiosa } & $\mathrm{Si}$ & 82 & 86,3 \\
\hline & No & 13 & 13,7 \\
\hline & Faltantes & 8 & 7,8 \\
\hline & Católica & 66 & 2,0 \\
\hline & Cristiana & 11 & 78,6 \\
\hline & Ninguna & 5 & 13,1 \\
\hline & Judía & 1 & 6 \\
\hline & Mormón & 1 & 1,2 \\
\hline & Faltantes & 19 & 1,2 \\
\hline \multirow{5}{*}{ Tipo de Familia } & Nuclear & 47 & 46,1 \\
\hline & Uniparental & 30 & 29,4 \\
\hline & $\operatorname{Re}$ & 14 & 13,7 \\
\hline & Extendida & 11 & 10,8 \\
\hline & Faltantes & 1 & 1 \\
\hline
\end{tabular}

Resultados de los cuestionarios antes del proceso educativo

\begin{tabular}{lccc}
\hline Variable & Niveles & N & $\%$ \\
\hline $\begin{array}{l}\text { Factor de riesgo } \\
\text { suicida }\end{array}$ & No & 59 & 57,3 \\
\cline { 2 - 4 } $\begin{array}{l}\text { (cuestionario de } \\
\text { Plutchick) }\end{array}$ & Si & 44 & 42,7 \\
\hline \multirow{2}{*}{$\begin{array}{l}\text { Cuestionario de } \\
\text { Autoestoestima } \\
\text { (Rosenberg) }\end{array}$} & Baja & 58 & 56,9 \\
\cline { 2 - 4 } & Normal & 37 & 36,3 \\
\cline { 2 - 4 } & Alta & 7 & 6,9 \\
\hline \multirow{2}{*}{$\begin{array}{l}\text { Escala dimensional } \\
\text { del sentido de la } \\
\text { vida } \\
\text { (Rathaus) }\end{array}$} & Asertividad & 1 & 1 \\
\cline { 2 - 4 } & Inasertivo & 11 & 10,7 \\
\cline { 2 - 4 } & Asertivo & 10 & 9,7 \\
\cline { 2 - 4 } & Minasertivo & 7 & 6,8 \\
\cline { 2 - 4 } & Masertivo & 4 & 3,9 \\
\hline \multirow{2}{*}{$\begin{array}{l}\text { Cuestionario Mito } \\
\text { o realidad }\end{array}$} & Promedio & 2,9 & \\
\cline { 2 - 4 } & Des. Est. &, 86 & \\
\cline { 2 - 4 } & LC95\%LI & 2,69 & \\
\cline { 2 - 4 } & LC95\%LS & 3,03 & \\
\hline
\end{tabular}

Resultados de los cuestionarios después del proceso educativo

\begin{tabular}{lccc}
\hline $\begin{array}{l}\text { Factor de riesgo } \\
\text { suicida } \\
\text { (cuestionario de }\end{array}$ & No & 96 & 93,2 \\
\cline { 2 - 4 } $\begin{array}{l}\text { Plutchick) } \\
\text { Cuestionario de }\end{array}$ & $\mathrm{Si}$ & 7 & 6,8 \\
\cline { 2 - 4 } $\begin{array}{l}\text { Autoestoestima } \\
\text { (Rosenberg) }\end{array}$ & Baja & 46 & 45,1 \\
\cline { 2 - 4 } & Normal & 46 & 45,1 \\
\cline { 2 - 4 } & Flta & 10 & 9,8 \\
\hline & Asertividad & 46 & 44,7 \\
\cline { 2 - 4 } $\begin{array}{l}\text { Escala dimensional } \\
\text { del sentido de la } \\
\text { vida } \\
\text { (Rathaus) }\end{array}$ & Inasertivo & 28 & 27,2 \\
\cline { 2 - 4 } & Asertivo & 28 & 27,2 \\
\cline { 2 - 4 } & Minasertivo & 1 & 1 \\
\cline { 2 - 4 } & Masertivo & 2,85 & \\
\hline & Promedio & 4,55 & \\
\cline { 2 - 4 } $\begin{array}{l}\text { Cuestionario Mito } \\
\text { o realidad }\end{array}$ & Des. Est. &, 510 & \\
\cline { 2 - 4 } & LC95\%LI & 4,45 & \\
\cline { 2 - 4 } & LC95\%LS & 4,65 & \\
\hline & & & 1 \\
\hline
\end{tabular}

Fuente: elaboración propia 


\section{Evaluación del proceso educativo}

El proceso educativo se evaluó mediante el procedimiento estadístico de prueba $\mathrm{t}$ para muestras apareadas, y se aplicó a los 5 cuestionarios empleados. La Tabla 2 presenta los resultados de esta evaluación, y se observa que en todos los casos la diferencia entre el pos-test y el pre-test resultó significativa $(p<0,001)$.

Tabla 2.

Evaluación del proceso educativo sobre factor de riesgo suicida en población de estudiantes de secundaria.

\begin{tabular}{|c|c|c|c|c|c|c|}
\hline Cuestionario & Prueba & Media & $\mathrm{n}$ & Des. Est. & $\begin{array}{l}\text { Error típ. } \\
\text { de la media }\end{array}$ & $\mathrm{p}$ \\
\hline \multirow{2}{*}{ Puntaje cuestionario Plutchick } & Post & 2,67 & 103 & 2,44 & ,24 & \multirow{2}{*}{, 000} \\
\hline & Pre & 5,76 & 103 & 3,46 & ,34 & \\
\hline \multirow{2}{*}{ Puntaje cuestionario Rosenberg } & Post & 34,41 & 103 & 4,47 & ,44 & \multirow{2}{*}{, 000} \\
\hline & Pre & 25,85 & 103 & 6,28 & ,62 & \\
\hline \multirow{2}{*}{$\begin{array}{l}\text { Puntaje cuestionario mito y } \\
\text { realidad }\end{array}$} & Post & 4,55 & 103 &, 51 & ,05 & \multirow{2}{*}{, 000} \\
\hline & Pre & 2,86 & 103 & ,85 & ,08 & \\
\hline \multirow{2}{*}{ Puntaje cuestionario Rathaus } & Post & 17,93 & 103 & 17,52 & 1,73 & \multirow{2}{*}{, 000} \\
\hline & Pre & $-2,52$ & 103 & 16,81 & 1,66 & \\
\hline \multirow{2}{*}{ Puntaje cuestionario Rathaus } & Pre & 2,54 & 103 & ,45 & ,044 & \multirow{2}{*}{,000 } \\
\hline & Post & 2,85 & 103 & ,33 & ,03 & \\
\hline
\end{tabular}

Fuente: elaboración propia

Mediante la prueba de $\mathrm{x}^{2}$ se cruzaron las categorizaciones para pre y pos-prueba del cuestionario de Plutchick $(p=0,017)$, el de Rosenberg $(p=0,071)$ y el de Rathaus $(p=0,004)$.

La Figura 1 despliega la relación para el cuestionario de Plutchik. Esta figura muestra que de los que tenían $\mathrm{FR}$ positivo (sí) $86,36 \%$ pasaron a tener FR negativo después del proceso, y de los que tenían FR negativo el 1,69\% pasaron a tenerlo positivo. La Figura 2 muestra lo propio para el cuestionario de Rathaus.
Figura 1.

Relación entre factor de riesgo suicida según Plutchick antes y después del proceso educativo.

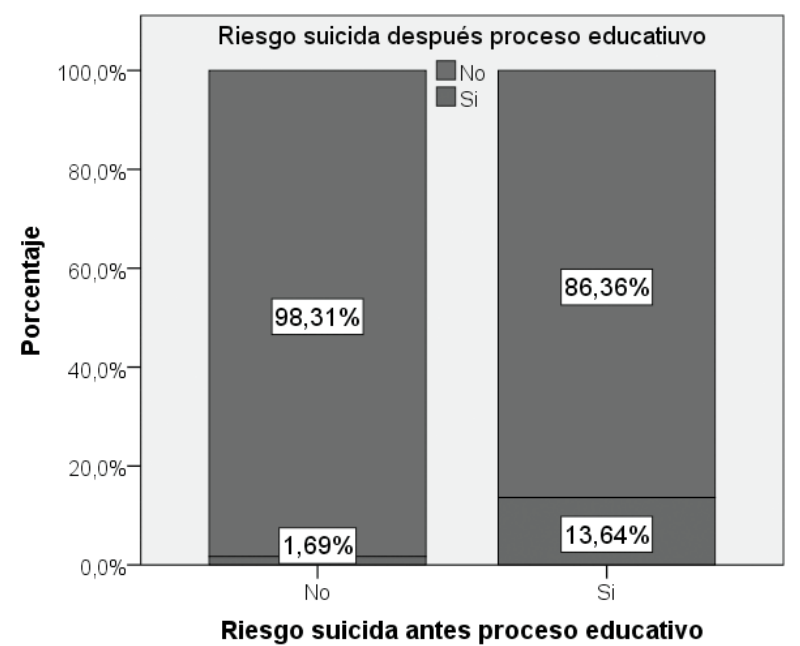

Fuente. Elaboración propia. 
Figura 2.

Relación entre el cuestionario de asertividad de Rathaus antes y después del proceso educativo.

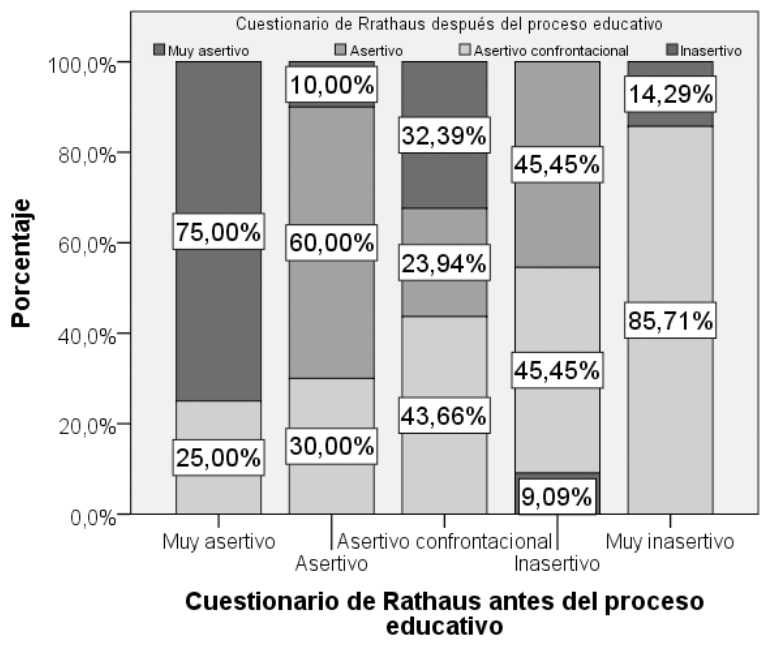

Fuente. Elaboración propia.

\section{Discusión}

En la investigación participaron 103 estudiantes de los grados $6^{\circ}$ a $8^{\circ}$ de un colegio de secundaria situado en la ciudad de Manizales (Colombia), donde el $53,9 \%$ de la población pertenece al género femenino; $37,4 \%$ a un estrato socioeconómico 2, y el promedio de edad es de 14,78 años (IC 95\%:14,5-15).

Al comenzar la investigación, en la realización del pre-test se detectó un factor de riesgo suicida positivo según cuestionario de Plutchick de $42,7 \%$, $56,9 \%$ tenían autoestima baja según el cuestionario de autoestima de Rosenberg, de acuerdo con la escala dimensional del sentido de la vida de Rathaus el $68,9 \%$ presentaron asertividad confrontativa. Referente al cuestionarlo mito o realidad que muestra ideas acertadas sobre el suicidio el promedio obtenido fue de 2,9 (IC 95\%: 2,69-3,03), donde 5 representa las ideas más acertadas. Posteriormente después del proceso de enseñanza, en el pos-test, los resultados obtenidos fueron: factor de riesgo suicida positivo de $6,8 \%$, en mayor proporción autoestima alta en $45,1 \%$, en mayor proporción $44,7 \%$ presentaron asertividad confrontativa. La escala dimensional del sentido de la vida presentó un promedio de 2,85 (IC 95\%:
2,79-2,02). El cuestionario mito o realidad presentó un resultado de 4,5 (IC 95\%: 4,45-4,65). El proceso de aprendizaje fue significativo $(p<0,001)$ y bastante exitoso. En particular el FR suicida pasó de un $42,7 \%$ (excesivo) a un 6,8\%, por debajo del nivel medido en estudios anteriores efectuados en colegios de secundaria de la ciudad de Manizales (Colombia) (Castaño-Castrillón, et al.,2015; Fuentes, et al., 2009).

Hay un amplio acuerdo entre profesionales sobre la importancia que tiene la prevención de la conducta suicida en general y se encuentran muchos documentos al respecto (Euregenas, 2013; OMS, 2001; OMS, 2012; OMS, 2014; Vargas, et al., 2015;). Sin embargo, son pocos los programas específicos de prevención del suicidio, que se encuentran referenciados al detalle, con reporte de resultados en la literatura científica, y dirigidos a población de colegios de secundaria. Bustamante y Florenzano (2013), efectuaron una amplia revisión de los programas de prevención del suicidio adolescente en establecimientos escolares, con resultados positivos reportados hay programas desarrollados en otros países que en general consisten en intervenciones mediante sesiones directas de trabajo con los estudiantes: "Surviving the teens", "Adolescents Depression Awareness Program (ADAP)", "Signs of Suicide (S.O.S)", "Care, Assess, Respond, Empower (CARE)", "Teen Screen", "National Youth Suicide Prevention Strategy" en Australia, "Gatekeepers", en Canadá, "Source of Strength", "Programa de Prevención del Suicidio" en Finlandia, "European Alliance Against Depression" (EAAD), son muy pocos los países que han dedicado un esfuerzo serio a este importante tema, en particular en Colombia no se encuentra nada al respecto.

Chávez, et al. (2008) reportan un programa para la prevención del suicidio en jóvenes, que consiste fundamentalmente en un taller (denominado "Salvando Vidas", de 10 horas en 5 sesiones), efectuado en preparatorias de la Universidad de Guanajuato (México). Al final del taller evaluaron fundamentalmente conocimientos sobre el tema, y comprobaron una mejora significativa en este aspecto. También, análogo a la presente investigación, se removieron mitos sobre el suicidio, pero no efectuaron ninguna evaluación referente 
al FR suicida, como si se hizo en la presente investigación. González y Gimeno (2013) reportan un programa educativo para la mejora de la autoestima en jóvenes escolares de España, y reportan una mejora fundamental de la autoestima luego del proceso. King, et al. (2011), reportan resultados preliminares del programa de prevención del suicidio denominado "Surviving the teens" $®$ efectuado en 1030 estudiantes de la ciudad de Cincinati (Estados Unidos), emplearon un instrumento desarrollado por los autores que comprendía, variables demográficas y diferentes aspectos de la conducta suicida. Similarmente a la presente investigación este instrumento se aplicó un día antes de la implementación del "programa educativo", un día después de terminarlo, y tres meses después, encontrando que después de 3 meses de seguimiento era menos probable que los estudiantes consideraran conductas suicidas que en el momento del pre-test. También buscaban ayuda más fácilmente, y era más probable que encontraran un adulto al cual consultar sus problemas; 9 de cada 10 pensaban que el programa debería ser replicado en otras escuelas. Sobre el "programa educativo" no mencionan absolutamente nada.

En Colombia se encuentra reportado un programa de intervención educativa en la temática del suicidio realizado por Piedrahita, Paz y Romero (2012), en 30 estudiantes de una institución educativa de la ciudad de Cali. El programa se realizó en 4 fases y estaba dirigido a identificar factores de riesgo suicida en la población participante, y efectuar un proceso educativo sobre conductas suicidas, mitos, etc. Finalmente, el $86 \%$ de los participantes del estudio manifestaron reconocer a las personas que se encuentran en riesgo de intento de suicidio, describieron cuando éstas necesitan que se les escuche, tener diálogo, recibir ayuda, no ser juzgada por sus acciones y ser remitida a un centro de salud. A diferencia de la presente investigación en este estudio no se determinó el FR suicida de los participantes.

En definitiva, la presente investigación fue idónea, para la población adolescentes, porque permitió el reforzamiento positivo, pudiendo corregir conductas autolesivas, y cambiando el concepto de suicidio como un tema tabú, resolviendo mitos e ideas equivocadas sobre este hecho. Al implementar el proyecto en este plantel se logró de manera eficiente erradicar los mitos y falacias que rodean esta población, se genera conciencia, conocimiento y una oportuna inter-vención en cuanto a estas prácticas y planeación nociva. Referente a la conducta suicida se deben valorar unos eventos ligados a: estados de angustia o de pánico, incapacitantes o dolorosas del adolescente, conflictos y disfunción familiar, deficientes redes de apoyo social y amistad, maltrato doméstico y abuso sexual, consumo de sustancias ilícitas; sumado a esto existen otras variables tales como son la edad, sexo, diferentes trastornos psiquiátricos y alteraciones psicológicas como la depresión mayor, trastornos afectivos y trastornos de ansiedad. Acentuando el valor del comportamiento suicida, se encuentran los sentimientos de desesperación, inutilidad, fracaso desasosiego, impotencia, resignación y frustración; estos componentes y otros más son los que llevan al adolecente a buscar "soluciones inmediatas", como el suicidio. No obstante, pese a estos compontes no todas las tentativas de suicidio conllevan al fallecimiento; un alto porcentaje de estos actos terminan en hospitalizaciones con secuelas a corto, mediano y largo plazo, lo más importante es que reciban el apoyo y acompañamiento suficiente para superar esos momentos de angustia (Vargas, et al., 2015).

Esta investigación induce a reducir la mayoría de casos posibles, de manera que se recomienda que los planteles educativos dispongan de personal capacitado para el reconocimiento de los factores de riesgo, ya sean consejeros escolares, guías de prevención del suicidio de este contribuyendo a detectar rápidamente a los estudiantes con ideación suicida, con el propósito de intervenir y reorientar al adolescente en riesgo, de tal manera que se pueda disminuir éste problema de salud pública. Justificándose esto por el hecho de que cada año incrementan la cantidad de jóvenes con ideación suicida y crece el fenómeno en las escuelas.

Para lo anterior, los métodos tenidos en cuenta la presente investigación (socializaciones, dibujos, expresión corporal) mostraron ser la mejor 
estrategia para fortalecer los factores protectores, generar en la población asertividad y resolución de conflictos, tal como lo recomienda la OMS en la estrategia de prevención del suicidio (OMS, 2001). De igual manera es necesario trabajar conjuntamente con los padres de familia y con los estudiantes en el plantel educativo, mediante talleres que permitan restablecer la autoestima, la autoimagen, el autocuidado; creando espacios para la reflexión y la expresión de sus emociones, que permitan adquirir herramientas sociales y destrezas intelectuales necesarias para afrontar las dificultades del día a día. Es importante hacer esta intervencón en los planteles educativos, ya que allí permanecen los jóvenes la mayor parte del tiempo.

Este proceso educativo expuesto en esta investigación mostró ser altamente exitoso, disminuyendo dramáticamente el FR suicida en estos estudiantes, mejorando también notoriamente su autoestima, asertividad y quitando mitos sobre el suicidio. Los resultados de la pre-prueba mostraron que esta población es de alto riego referente a conductas suicidas y que una intervención puede ser altamente exitosa. Sin embargo, una sola intervención piloto como la acá descrita no es suficiente (se sabe que después de un tiempo de la intervención, si no hay refuerzos, la población vuelve a su estado inicial). Es necesario implementar en estas poblaciones escolares de alto riesgo, procesos educativos como el acá mostrado, pero que muestren continuidad en el tiempo, que sea un apoyo permanente de las instituciones educativas a sus estudiantes. Los directivos y docentes de instituciones escolares deberían mostrar más interés por procesos de apoyo a sus estudiantes, teniendo en cuenta que comúnmente es población de alto riesgo. Además estos programad deberían tener un decidido apoyo estatal.

Durante la ejecución del proyecto se identificaron las siguientes limitaciones: ausencia de los participantes, debido a la deserción escolar o desinterés de la población objetivo. Múltiples responsabilidades académicas lo cual disminuyó el tiempo disponible para interactuar con los estudiantes. Debilidades en la coordinación y compromiso por parte del personal docente para realizar las reu- niones con los estudiantes; dado que en algunas ocasiones coincidió con actividades de refuerzo académico y "universidad en tu colegio". Falta de compromiso del cuerpo directivo y docente de la institución educativa ya que se incumplió con sesiones programadas. Escases de recursos financieros para el desarrollo de las actividades.

\section{Referencias}

Atienza, F. L., Moreno, Y., \& Baaguer, I. (2000). Análisis de la dimensionalidad de la Escala de Autoestima de Rosenberg en una muestra de adolecentes valencianos. Revista de Psicología. Universitas Tarraconensis, 22, 29-42.

Bustamante, F., \& Florenzano, R. (2013). Programas de prevención del suicidio adolescente en establecimientos escolares: una revisión de la literatura. Revista Chilena de Neuropisuiatría, 51(2), 126-136.

Castañeda, G.M. (2014). La biodanza como práctica corporal En relación con la promoción de la salud. Revista Educación física y deporte, 28, 81-90.

Castaño-Castrillón, J.J., Cañón, S.C., BetancurBetancur, M., Castellanos-Sánchez, P.L., Castellanos-Sánchez, P.L., Guerrero-González, J. \& Llanos-Calderón, C. (2015). Factor de riesgo suicida según dos cuestionarios, y factores asociados en estudiantes de la universidad nacional de Colombia sede Manizales. Diversitas: perspectivas en psicología, 11(2), 193-205.

Chávez, A.M., Medina, M.C., \& Macías, L.F. (2008). Modelo psicoeducativo para la prevención del suicidio en jóvenes. Salud Mental, 31, 197-203.

Durkheim, E. (1992). El Suicidio. México DF: Editorial Fontamara.

Euregenas. (2013). Directrices Generales para la Prevención del Suicidio. Gante: Euregenas.

Fuentes, M.M., González, A., Castaño Castrillón, J.J., Hurtado, C.F., Ocampo, P.A., Páez Cala, M.L., Zuluaga, L.M. (2009). Riesgo suicida y 
factores relacionados en estudiantes de $6^{\circ}$ a $11^{\circ}$ grado en colegios de la ciudad de Manizales (Colombia). 2007-2008 Archivos de Medicina (Manizales), 9, 2,110-122.

González, F., \& Gimeno, A. (2013). Menores en situación de riesgo social: Valoración de un programa para la mejora de la autoestima. Psychosocial Intervention, 22, 1-5.

Gutiérrez, E. (2010). El acoso psicológico en el trabajo y su impacto en el clima laboral, en una organización educativa y otra de salud. (Tesis de doctorado). Santiago de Querétaro: Universidad Autónoma de Querétaro, Facultqad de Contaduría y Administración.

Henao, R., López, D., \& Mosquera, O. (2014). Narrativas de jóvenes sobre los sentidos de convivencia pacífica en la escuela. (Tesis de Maestrìa). Manizales: Universidad de Manizales-CINDE..

Herrera, P.M., \& Betancour, K.A. (2016). Factores familiares de riesgo en el intento suicida. Revista Cubana de Medicina General Integral, 16, 134-137.

Instituto Nacional de Estadísticas y Geografía. (2015). Estadisticas a propósito del día mundial para la prevención del suicidio. Aguascalientes: Instituto Nacional De Estadística y Geografía de México.

King, K.A., Strunk, C.M., y Sorter, M. T. (2011). Preliminary effectiveness of surviving the teens( $\left({ }^{\circledR}\right)$ suicide prevention and depression awareness program on adolescents' suicidality and self-efficacy in performing helpseeking behaviors. Journal of School Health, 81(9), 581-90.

León, M. (2014). Revisión de la escala de asertividad de Rathus adaptada por León y Vargas (2009). Reflexiones, 93, 157-171.

Londoño, C., \& Valencia, C. (2008). Asertividad, resistencia a la presión de grupo y consumo de alcohol en universitarios. Acta Colombiana de psicología, 11,155-162.
Macana, N.L. (2011). Comportamiento del suicidio en Colombia. Bogotá DC: Instituto Nacional de Medicina legal y Ciencias Forenses.

Martínez, E., Trujio, A. M., Díaz, J. P., \& Osma, J. J. (2011) Desarrollo y estructura de la escala dimensional del sentido de vida. Acta Combiana de psicología, 14, 113-119.

Nope, M.Y, (2015). Informe de seguridad en Bogotá Comportamiento de las muertes violentas. Bogotá DC: Veeduria Distrital.

Oiva, S. (2014). Dimensiones de sentitido de vida en estudiantes de la carrera de licenciatura en sicología clinica y licenciatura en sicología industrial/organizacional de 5to. Año de la Universidad Rafael Landívar. Guatemala de la Asunción: U .Rafael Landívar..

Organización Mundia de la Salud. (2001). Prevención del Suicidio un instrumento para docentes y demás personal institucional. Ginebra: Departamento de Salud Mental y Toxicomanías.

Organización Mundial de la Salud. (2012). Prevención del suicidio. Ginebra: Organización Mundial de la Salud.

Organización Mundial de la Salud. (2014). Prevención de suicidio un imperativo global. Washington DC: Organización panamericana de la salud.

Piedrahita, L.E., Paz, K.M., \& Romero, A.M. (2012). Estrategia de intervención para la revención del suicidio en adolescentes; la escuela como contexto. Hacia la Promoción de la Salud, 17. 136-148.

Pitman, A., Osborn, D., King, M., \& Erlangsen, A. (2014). Effects of suicide bereavement on mental health and suicide risk. The Lancet Psychiatry, 1(1), 86-94.

Plutchik, R., Van Praag, H.M. Conte, H.R., \& Picard, S. (1989). Correlates of Suicide and violent Risk, I: The suicide Risk Measure. Comprehensive Psychiatry, 30(4), 296-302. 
Rodríguez, C., \& Castaño, M. (2002). El test de Árbol. Su aplicación en la exploración de personalidad en la clínica médico forense. Cuaderno de medicina Forense, 30, 5-21.

Rojas, C.A., Zegers, B., \& Forster, C.E. (2009). La escala de autoestima de Rosenberg: Validación para Chile en una muestra de jóvenes adultos, adultos y adultos mayores. Revista Médica de Chile, 137, 791-800.

Rosenberg, M. (1965). Escala de autoestima de Rosenberg (RSE): La sociedad y la imagen de sí mismo adolescente. Princeton: University press.

Rubio, G., Montero, I., Jáuregui, J., Villanueva, R., Casado, M.A., Marín, J.J., \& Santo-Domingo, J. (1998). Validación de la escala de riesgo suicida de Plutchik en población española. Archivos de Neurobiología, 61, 143-52.
Ruiz, A. (2009). La relación entre el intento suicida y la conducta antisocial en una muestra de estudiantes en nivel medio y medio superior de la ciudad de México. México DF: Universidad Nacional Autónoma de México.

Salgado, I. (2011). Aprendizaje-enseñanza: mejora a partir de la interacción de los actores. Educación y educadores, 14, 457-474.

Vargas, G., Vásquez, C. F., Soto, G. M., \& Ramírez, L. M. (2015). Atención con calidad a las personas que presentan conducta suicida: manual para profesionales de la salud / Secretaría de Salud Alcaldía de Medellín. Medellín: Centro Nacer, Salud Sexual y Reproductiva / Biblioteca Electrónica.

Ventura, R., Carvajal, C., Undurraga, S., Vicuña, P., Egaña, J., \& Garib, M. J. (2010). Prevalencia de ideación e intento suicida en adoLescente en a región Metropolitana de Santiago de Chile. Revista Médica de Chile, 138, 309-315. 